\title{
SURGERY UP TO HOMOTOPY EQUIVALENCE FOR NONPOSITIVELY CURVED MANIFOLDS
}

\author{
A. NICAS AND C. STARK
}

\begin{abstract}
Let $M^{n}$ be a smooth closed manifold which admits a metric of nonpositive curvature. We show, using a theorem of Farrell and Hsiang, that if $n+k \geqslant 6$, then the surgery obstruction map $\left[M \times D^{k}, \partial ; G / \mathrm{TOP}\right] \rightarrow L_{n+k}^{h}\left(\pi_{1} M, w_{1}(M)\right)$ is injective, where $L_{*}^{h}$ are the obstruction groups for surgery up to homotopy equivalence.
\end{abstract}

Farrell and Hsiang have shown that if $M^{n}$ is a closed nonpositively curved Riemannian manifold and $n+k \geqslant 6$, then the surgery map

$$
\left[M^{n} \times D^{k}, \partial ; G / \mathrm{TOP}, *\right] \stackrel{\theta_{s}}{\rightarrow} L_{n+k}^{s}\left(\pi_{1} M, w_{1}(M)\right)
$$

for topological surgery in a split monomorphism [1]. This note considers the implications of their result of $h$-surgery, i.e. surgery up to homotopy equivalence rather than simple homotopy equivalence.

LEMma. Let $Q^{p}$ be a compact manifold such that $\theta_{s}:\left[Q^{p}, \partial ; G / \mathrm{TOP}, *\right] \rightarrow$ $L_{p}^{s}\left(\pi_{1} Q, w_{1}(Q)\right)$ is monic, let $N^{n}$ be a compact, connected submanifold of $Q^{p}$ which has a normal microbundle $\nu_{Q}(N)$, and let $f: U^{p} \rightarrow Q^{p}$ be a simple homotopy equivalence which is a homeomorphism near the boundary. Then any normal map $\left.f\right|_{V}: V=f^{-1}(N)$ $\rightarrow N$ induced by making $f$ transverse to $N$ is normally cobordant to a homeomorphism.

Proof. Recall that the long exact sequence of topological surgery

$$
\begin{aligned}
\rightarrow \varsigma^{a}\left(Q^{p} \times D^{k}, \partial\right) & \stackrel{\eta_{a}}{\rightarrow}\left[Q^{p} \times D^{k}, \partial ; G / \mathrm{TOP}, *\right] \stackrel{\theta_{a}}{\rightarrow} L_{p+k}^{a}\left(\pi_{1} Q, w_{1}(Q)\right) \\
& \stackrel{\partial}{\rightarrow} \S^{a}\left(Q^{p} \times D^{k-1}, \partial\right) \stackrel{\eta_{a}}{\rightarrow} \cdots \rightarrow\left[Q^{p}, \partial ; G / \mathrm{TOP}, *\right] \stackrel{\theta_{a}}{\rightarrow} L_{p}^{a}\left(\pi_{1} Q, w_{1}(Q)\right)
\end{aligned}
$$

(where $a=s$ or $h$ denotes surgery up to simple homotopy equivalence or up to homotopy equivalence, respectively) is a long exact sequence of groups and group homomorphisms (see [4]; also see [2] for surgery in the topological category). Let $x=[f: U \rightarrow Q] \in \mathcal{S}^{s}(Q, \partial)$ and let $i: N \hookrightarrow Q$ be the inclusion map. Then the element $\left[\left.f\right|_{V}: \quad V \rightarrow N\right] \in[N, \partial ; G / \mathrm{TOP}, *]$ defined above is $i^{*}\left(\eta_{s}(x)\right)$, where $i^{*}$ : $[Q, \partial ; G / \mathrm{TOP}, *] \rightarrow[N, \partial ; G / \mathrm{TOP}, *]$. Since $\theta_{s}$ is a monomorphism, image $\left(\eta_{s}\right)=0$ in $[Q, \partial ; G / \mathrm{TOP}, *]$, and since $i^{*}$ is a homomorphism of groups we see that

Received by the editors December 15, 1982 and, in revised form, August 15, 1983.

1980 Mathematics Subject Classification. Primary 57R67, 57R65.

1984 American Mathematical Society $0002-9939 / 84 \$ 1.00+\$ .25$ per page 
$i^{*}\left(\eta_{s}(x)\right)=i^{*}(0)=0$, or that $i^{*}\left(\eta_{s}(x)\right)$ is normally cobordant to a homeomorphism.

Geometrically, we have made the following construction. image $\left(\eta_{s}\right)=0$ implies that each element $x$ of the $s$-structure set $\delta^{s}(Q, \partial)$ is obtained from a homeomorphism of $Q$ by the action of an element of $L_{p+1}^{s}\left(\pi_{1} Q, w_{1}(Q)\right)$. That is, there is a normal map $\left[\left(X^{p+1}, \partial_{0} X, \partial_{1} X\right) \stackrel{g}{\rightarrow}(Q \times I, Q \times 0, Q \times 1)\right]$ so that $\left.g\right|_{\partial_{0}}: \partial_{0} X \rightarrow Q \times$ 0 is a homeomorphism and $\left.g\right|_{\partial_{1} X}: \partial_{1} X \rightarrow Q \times 1$ is a simple homotopy equivalence representing $x \in \mathcal{S}^{s}(Q, \partial)$. Make $g$ transverse to $N \times I \subset Q \times I$ and let $Y=$ $g^{-1}(N \times I)$. Then $\left[g^{-1}(N \times 1) \rightarrow N \times 1\right]$ represents $i^{*}\left(\eta_{s}(x)\right)$ and $\left.g\right|_{Y}: Y \rightarrow N \times I$ is the desired normal bordism.

Proposition. If $M^{n}$ is a closed, nonpositively curved manifold then $\theta_{h}$ : [M× $\left.D^{k}, \partial ; G / \mathrm{TOP}, *\right] \rightarrow L_{n+k}^{h}\left(\pi_{1} M, w_{1}(M)\right)$ is split monic if $n+k \geqslant 6$.

Proof. We may use the Lemma to show that $\theta_{h}$ is monic, i.e. that image $\left(\eta_{h}\right)=0$. Let $x \in \delta^{h}\left(M \times D^{k}, \partial\right)$ be represented by a homotopy equivalence $f:\left(V^{n+k}, \partial\right) \rightarrow$ $\left(M \times D^{k}, \partial\right)$ which is a homeomorphism near the boundary. Since taking products with the circle kills Whitehead torsion [3], $f \times \operatorname{Id}_{S^{1}}: V \times S^{1} \rightarrow M \times D^{k} \times S^{1}$ is a simple homotopy equivalence. Since $M$ is nonpositively curved, so is $M \times S^{1}$, and [1] shows that $\theta_{s}$ is monic for $M \times S^{1}$. Apply the Lemma with $Q=M \times S^{1}$ and $N=M \times 1$ to conclude that $f$ is normally cobordant to a homeomorphism, i.e. $\eta_{h}(x)=0$.

The splitting in [1] of $\theta_{s}$ induces a splitting of $\theta_{h}$ if we consider this commutative diagram:

$$
\begin{array}{ccc}
{\left[M^{n}, \partial ; G / \mathrm{TOP}, *\right]} & \stackrel{\theta_{h}}{\rightarrow} & L_{n}^{h}\left(\pi_{1} M, w_{1}(M)\right) \\
p^{*} \downarrow & & \downarrow \operatorname{tr}(p) \\
{\left[M^{n} \times S^{1}, \partial ; G / \mathrm{TOP}, *\right]} & \stackrel{\theta_{s}}{\rightarrow} & L_{n+1}^{s}\left(\pi_{1} M \times \mathbf{Z}, w_{1}(M) \circ \mathrm{pr}_{1}\right)
\end{array}
$$

Here $p: M^{n} \times S^{1} \rightarrow M^{n}$ is a first-coordinate projection and $\operatorname{tr}(p)$ is the transfer associated to $p[4, \S 4.2]$. (The effect of $\operatorname{tr}(p)$ on one of the "objects" used in defining $L_{n}^{h}\left(\pi_{1} M, w_{1}(M)\right)$ in [5, Chapters 9 and 17D] is to multiply a map $(B, A) \rightarrow(Y, X)$ inducing a homotopy equivalence $A \rightarrow X$ by $\operatorname{Id}_{S^{1}}$; by [3] this gives a map ( $B \times S^{1}, A$ $\left.\times S^{1}\right) \rightarrow\left(Y \times S^{1}, X \times S^{1}\right)$ inducing a simple homotopy equivalence $A \times S^{1} \rightarrow X$ $\times S^{1}$, i.e. an "object" for the definition of $L_{n+1}^{s}\left(\pi_{1} M \times \mathbf{Z}, w_{1}(M) \circ \mathrm{pr}_{1}\right)$.) If $i$ : $M \rightarrow M \times S^{1}$ is the inclusion map, then $i^{*}$ splits $p^{*}$. Compose $i^{*}$ with the splitting of [1] for $\theta_{s}$ and with $\operatorname{tr}(p)$ to split $\theta_{h}$.

More generally, the Proposition holds for any closed manifold $M^{n}$ such that $M^{n}$ and $M^{n} \times S^{1}$ both satisfy condition (*) of [1, p. 201].

ACKNOWLEDGEMENT. We wish to thank the referee for a helpful remark.

\section{REFERENCES}

1. F. T. Farrell and W. C. Hsiang, On Novikov's conjecture for non-positively curved manifolds. I, Ann. of Math. (2) 113 (1981), 199-209.

2. R. C. Kirby and L. C. Siebenmann, Foundational essays on topological manifolds, smoothings, and triangulations, Princeton Univ. Press, Princeton, N. J., 1977. 
3. K. W. Kwun and R. H. Sczarba, Product and sum theorems for Whitehead torsion, Ann. of Math. (2) 82 (1965), 183-190.

4. A. J. Nicas, Induction theorems for groups of homotopy manifold structures, Mem. Amer. Math. Soc. No. 267 (1982).

5. C. T. C. Wall, Surgery on compact manifolds, Academic Press, New York, 1970.

Department of Mathematics, University of Toronto, Toronto, Ontario, Canada M5S 1A1

Department of Mathematics, Brandeis University, Waltham, Massachusetts 02254 